Article

\title{
A Resource Allocation Scheme for Multi-D2D Communications Underlying Cellular Networks with Multi-Subcarrier Reusing
}

\author{
Yong Zhang ${ }^{1}$, Fangmin $\mathrm{Li}^{1,2}$, Mohammed Abdulaziz Aide Al-qaness ${ }^{1}$ and Xidao Luan ${ }^{2, *}$ \\ 1 School of Information Engineering, Wuhan University of Technology, Wuhan 430070, China; \\ zhang_yong@whut.edu.cn (Y.Z.); lifangmin@whut.edu.cn (F.L.); alqaness@whut.edu.cn (M.A.A.A.-q.) \\ 2 Department of Mathematics and Computer Science, Changsha University, Changsha 410022, China \\ * Correspondence: Xidaoluan@ccsu.cn; Tel.: +86-731-8426-1249
}

Academic Editor: Elli Kartsakli

Received: 12 December 2016; Accepted: 25 January 2017; Published: 7 February 2017

\begin{abstract}
Device-to-device (D2D) communication is proposed as a promising technique of future cellular networks which fulfills its potential in terms of high resource utilization. In this paper, in order to improve the achievable rate of D2D communication and the spectrum utilization, we consider the scenario that multiple D2D pairs can share uplink spectrum resources with multiple cellular users (CUs). We aim to maximize the overall system spectrum efficiency while satisfying the rate requirements of all CUs and guaranteeing that the system gain is positive. We formulate the joint optimization problem of subcarrier assignment and power allocation which falls naturally into a mixed integer non-linear programming form that is a difficult problem to solve. Hence, we propose a two-stage resource allocation scheme which comprises a subcarrier assignment by employing a heuristic greedy strategy, as well as a power allocation algorithm based on the Lagrangian dual method. Numerical results demonstrate the advantageous performance of our scheme in greatly increasing the system sum spectrum efficiency.
\end{abstract}

Keywords: cellular network; multiple device-to-device (D2D) communication; mixed integer non-linear programming; multi-subcarrier reusing; power allocation

\section{Introduction}

Device-to-device (D2D) communication has been proposed as an underlay to cellular networks where user equipments (UEs) in proximity are able to communicate directly with one another with the support of evolved node B (eNB) [1,2]. D2D communication pairs bring a large amount of benefits for users, such as expanding the service coverage area, increasing data rate, improving system energy efficiency, enhancing network throughput, reducing network delay, creating new types of services, and so on [3,4]. Additionally, the spectral efficiency could be further improved by allowing D2D users (DUs) to share the same spectrum resources with cellular users (CUs). In this case, intra-cell interference between DUs and CUs is no longer negligible so that a proper radio resource allocation strategy is needed to obtain the potential performance gains.

Most of the existing works, so far, have been carried out on resource allocation for hybrid D2D-cellular systems in order to improve spectrum efficiency and mitigate the interference between D2D and cellular communications. In [5], an interference limited area was defined for uplink underlaying systems, where no CUs can reuse the same spectrum resource with D2D pair to avoid strong interference. Various resource reusing ways are considered in [6], where eNB can allocate either orthogonal or non-orthogonal radio resources to CUs and D2D users. A new spectrum sharing protocol for D2D communication overlaying a cellular network is proposed in [7], which allows the D2D users 
to act as a relay to assist the two-way communications between the eNB and the CUs. The authors of [8] developed an optimal and low-complexity channel reusing algorithm to maximize the number of admitted D2D pairs. The research in [9] focuses on resource allocation and interference management issue in D2D communication underlaying cellular networks, where the system sum-rate optimization for D2D and cellular links is considered. Both the works of Yu et al. and Jung et al. [10,11] consider the joint mode selection and resource allocation problem that aims to improve the sum-rate and the power efficiency. While guaranteeing the quality of service (QoS) of both CUs and D2D pairs, a centralized spectrum allocation scheme has been designed to maximize the system throughput in [12]. In [13], a coalitional game approach is employed to solve the joint mode selection and D2D resource allocation.

The aforementioned works generally focus on investigating the resource allocation in the case that one D2D pair could reuse only one cellular resource, even though there are much more CUs in practical networks. Actually, the system performance parameters, such as spectral efficiency, will be further improved if a D2D pair could reuse the resources of multiple CUs. Since more subcarriers are available, the DUs would have more opportunities to explore the multi-channel diversity, thereby making spectrum utilization more efficient. There are some works on resource allocation for multicarrier optimization problem in different systems. In [14,15], the authors propose a resource allocation algorithm design to iteratively find the optimal solution for maximization of the energy efficiency of orthogonal frequency division multiple access (OFDMA) systems based on the Dinkelbach transformation. In [16], a resource allocation algorithm where D2D links can reuse the resources of more than one CU is proposed, while the QoS is not guaranteed for CUs. The authors of [17] study optimal and suboptimal power allocation methods such that the D2D link can maximally utilize multiple possible cellular resources and meanwhile the QoS of CUs is guaranteed, however, they only considered a simple scenario where there only one D2D pair exists.

In this paper, we consider a system where multiple D2D links reuse the uplink resources of multiple CUs. We formulate the problem as a mixed integer non-linear programing (MINLP) problem with the objective to maximize the spectrum efficiency of the heterogeneous network. Due to the high complexity of original problem, we decompose it into two cascaded subcarrier assignment and power allocation problems. While the former is to assign subcarriers for D2D pairs by a heuristic algorithm, the latter can be solved by a power allocation algorithm based on the Lagrangian dual method. Specifically, our work makes the following contributions: (1) We investigate the optimal resource allocation for the case that one D2D pair could reuse more than one subcarrier while guaranteeing the minimum rate requirements of individual CUs; (2) We introduce the system positive gain and the system negative gain which represent the benefit of reusing cellular resources and the penalty for interference exerted on CUs respectively. The network final profit should be guaranteed (i.e., the overall system gain should be positive); (3) We develop a greedy-based algorithm to allocate one or more subcarriers to each D2D pair, as well as an efficient power allocation algorithm. The proposed resource allocation scheme can significantly improve the sum spectrum efficiency of the overall system.

The rest of the paper is organized as follows. In Section 2, we describe the system model and problem formulation. In Section 3, we derive and propose the two-stage algorithm to solve the resource allocation problem, and simulation results are presented and discussed in Section 4. Finally, Section 5 concludes the paper.

\section{System Model and Problem Formulation}

\subsection{System Model}

To make this paper easy to follow, we present some frequently used notations in the considered system model in Table 1. 
Table 1. Symbol Notations. CU, cellular users; eNB, evolved node B; D2D, device-to-device.

\begin{tabular}{cl}
\hline Symbol & The set of the CUs \\
$M$ & The number of the CUs \\
$p_{m}^{C}$ & The transmission power of the CU $m$ \\
$h_{m, B}^{C B}$ & The channel gain from the CU $m$ to the eNB on the $m$ th subcarrier \\
$R_{m}^{C}$ & The spectrum efficiency of CU $m$ without resource sharing \\
$R_{m, \text { min }}^{C}$ & The spectrum efficiency requirement of the CU $m$ \\
$\mathcal{K}$ & The set of the D2D pairs \\
$K$ & The number of the D2D pairs \\
$p_{k, m}^{D}$ & The transmission power of the D2D-Tx $k$ on the mth subcarrier \\
$P_{\text {thr }}^{D}$ & The maximum transmission power of the D2D-Tx \\
$h_{k}^{D D}$ & The channel gain from the D2D-Tx $k$ to the D2D-Rx $k$ \\
$h_{k}^{D B}$ & The channel gain from the D2D-Tx $k$ to the eNB \\
$h_{k, m}^{C D}$ & The channel gain from the CU m to the D2D-Rx $k$ \\
$R_{k, m}^{C}\left(p_{k, m}^{D}\right)$ & The spectrum efficiency of the CU $m$ with resource sharing \\
$R_{k, m}^{D}\left(p_{k, m}^{D}\right)$ & The spectrum efficiency of the D2D pair $k$ with resource sharing \\
$\Delta R_{m}^{C}\left(p_{k, m}^{D}\right)$ & The spectrum efficiency decrement of the CU $m$ \\
$\Delta R_{k, m}^{D}\left(p_{k, m}^{D}\right)$ & The spectrum efficiency gain of the D2D pair $k$ \\
$\sigma_{0}^{2}$ & The noise power on each subcarrier \\
$G_{k, m}^{P}\left(p_{k, m}^{D}\right)$ & The system positive gain \\
$G_{k, m}^{N}\left(p_{k, m}^{D}\right)$ & The system negative gain \\
$\pi_{k, m}$ & The D2D pairs assignment index \\
$\mathcal{M}_{k}$ & The set of the subcarriers which are assigned to the $k$ th D2D pair \\
\hline
\end{tabular}

As shown in Figure 1, we consider an uplink heterogeneous network underlaid with $K$ D2D pairs and $M$ CUs, where $K<M$. Let $\mathcal{K}=\{1,2, \ldots, K\}$ and $\mathcal{M}=\{1,2, \ldots, M\}$ be the sets of the D2D pairs and the CUs, respectively. We assume that the overall radio spectrum is divided into $M$ subcarriers, or resource blocks (RBs) or chunks [18-20]. Each CU uses one of the subcarriers with a specific transmit power. Thus, there is no vacant spectrum and a fully loaded cellular network scenario is investigated similarly to [9]. The eNB can obtain the instantaneous channel state information (CSI) on all involved links. In order to improve the achievable rate of D2D users, the D2D pairs are allowed to reuse multiple cellular uplink resources for direct transmission.

The channel gain from the $\mathrm{CU} m$ to the eNB is denoted by $h_{m, \mathrm{~B}}^{\mathrm{CB}}$, and the channel gain from the $\mathrm{CU}$ $m$ to the D2D receiver (D2D-Rx) $k$ by $h_{k, m}^{C D}$. The channel gain from the D2D transmitter (D2D-Tx) $k$ to its receiver is denoted by $h_{k}^{D D}$, and the channel gain from the D2D-Tx $k$ to the eNB by $h_{k, B}^{D B}$. Assuming that $\mathrm{D} 2 \mathrm{D}$ pair $k$ reuses the subcarrier of $\mathrm{CU} m$, the received signal of $\mathrm{CU} m$ at the eNB is

$$
y_{k, m}^{C}=h_{m, B}^{C B} x_{m}^{C}+h_{k, B}^{D B} x_{k}^{D}+n_{m}^{C}
$$

where $x_{m}^{C}$ and $x_{k}^{D}$ represent the transmit signal by CU $m$ and D2D pair $k$, respectively, and $n_{m}^{C}$ represents the additive white Gaussian noise (AWGN) with a variance of $\sigma_{0}^{2}$. Similarly, the D2D-Rx received signal on subcarrier $m$ is

$$
y_{k, m}^{D}=h_{k}^{D D} x_{k}^{D}+h_{k, m}^{D C} x_{m}^{C}+n_{k}^{D}
$$

where $n_{k}^{\mathrm{D}}$ represents the AWGN at the eNB with a variance of $\sigma_{0}^{2}$, and the received signal of CU $m$ at the eNB without resource reusing can be expressed as

$$
y_{k, m}^{C}=h_{m, B}^{C B} x_{m}^{C}+n_{m}^{C}
$$




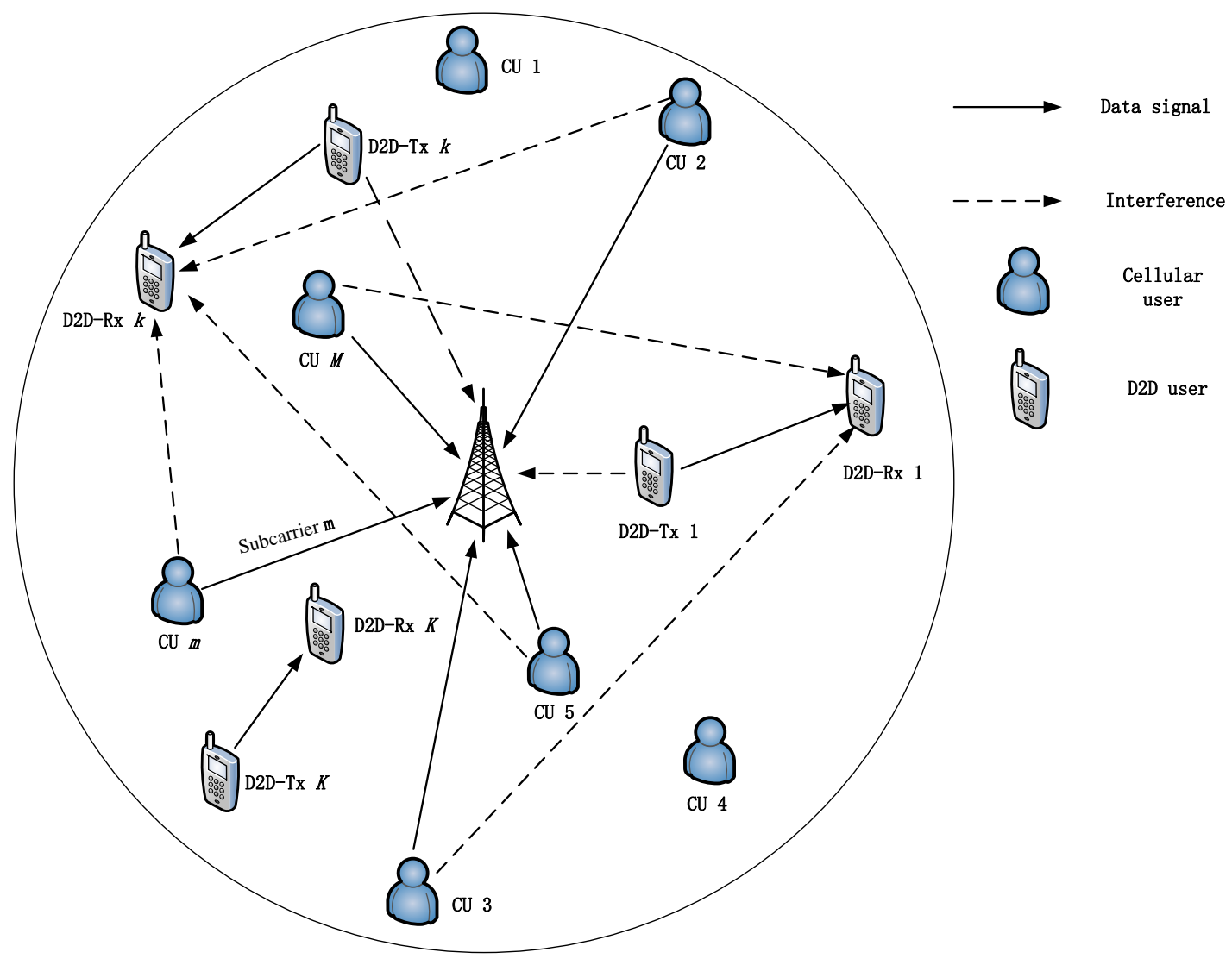

Figure 1. System model of D2D multicast groups and CUs.

Since the D2D link $k$ shares the same subcarrier with $\mathrm{CU} m$, they can generate mutual interference to each other. The channel capacities, i.e., the spectrum efficiency of the CU $m$ and the D2D pair $k$, can be respectively expressed as

$$
\begin{gathered}
R_{k, m}^{C}\left(p_{k, m}^{D}\right)=\log _{2}\left(1+\frac{p_{m}^{C} h_{m, B}^{C B}}{p_{k, m}^{D} h_{k, B}^{D B}+\sigma_{0}^{2}}\right) \\
R_{k, m}^{D}\left(p_{k, m}^{D}\right)=\log _{2}\left(1+\frac{p_{k, m}^{D} h_{k}^{D D}}{p_{m}^{C} h_{k, m}^{C D}+\sigma_{0}^{2}}\right)
\end{gathered}
$$

where $p_{m}^{C}$ and $p_{k, m}^{D}$ are the transmission power of the CU $m$ and D2D-Tx $k$ on the $m$ th subcarrier, respectively. Similarly, the spectrum efficiency of $\mathrm{CU} m$ without resource sharing can be expressed as

$$
R_{m}^{C}=\log _{2}\left(1+\frac{p_{m}^{C} h_{m, B}^{C B}}{\sigma_{0}^{2}}\right)
$$

By observing Equations (4) and (6), it is found that there is a decrement of the spectrum efficiency at $\mathrm{CU} m$, which is caused by the interference of the transmitter of D2D pair $k$. Thus, we have the spectrum efficiency decrement of the $\mathrm{CU} m$, i.e.,

$$
\Delta R_{m}^{C}\left(p_{k, m}^{D}\right)=R_{m}^{C}-R_{k, m}^{C}\left(p_{k, m}^{D}\right)
$$


Therefore, the spectrum efficiency gain after D2D pair $k$ reuses the subcarrier of CU $m$ is

$$
\begin{aligned}
\Delta R_{k, m}^{D}\left(p_{k, m}^{D}\right) & =R_{k, m}^{D}\left(p_{k, m}^{D}\right)-\Delta R_{m}^{C}\left(p_{k, m}^{D}\right) \\
& =R_{k, m}^{D}\left(p_{k, m}^{D}\right)+R_{k, m}^{C}\left(p_{k, m}^{D}\right)-R_{m}^{C}
\end{aligned}
$$

For the resource sharing between D2D pair $k$ and $\mathrm{CU} m$, it may bring the network additional benefit in signal-to-interference-plus-noise ratio (SINR); it also causes a loss of cellular system for the interference. We introduce the system positive gain and the system negative gain to express the benefit and the loss as $G_{k, m}^{P}$ and $G_{k, m}^{N}$, respectively. Thus, we can have

$$
\begin{gathered}
G_{k, m}^{P}\left(p_{k, m}^{D}\right) \triangleq \frac{p_{k, m}^{D} h_{k}^{D D}}{p_{m}^{C} h_{k, m}^{C D}+\sigma_{0}^{2}} \\
G_{k, m}^{N}\left(p_{k, m}^{D}\right) \triangleq \frac{p_{k, m}^{D} h_{k, \mathrm{~B}}^{D B}+\sigma_{0}^{2}}{\sigma_{0}^{2}}
\end{gathered}
$$

Therefore, upon achieving a positive system gain in resource allocation, we can have

$$
G_{k, m}^{P}\left(p_{k, m}^{D}\right) \geq G_{k, m}^{N}\left(p_{k, m}^{D}\right)
$$

\subsection{Problem Formulation}

In this paper, we aim to maximize the sum spectrum efficiency of the D2D pairs and CUs while guaranteeing a positive system gain and the rate requirement of CUs. Let $\pi_{k, m}=1$ denote the D2D pair $k$ reuse the subcarrier of $\mathrm{CU} m$, and $\pi_{k, m}=0$ otherwise. Mathematically, the considered resource allocation problem can be formulated as

$$
\max _{\left\{p_{k, m}^{\mathrm{D}}, \pi_{k, m}\right\}} \sum_{m=1}^{M}\left(R_{m}^{C}+\sum_{k=1}^{K} \pi_{k, m} \cdot \Delta R_{k, m}^{D}\left(p_{k, m}^{D}\right)\right)
$$

subject to

$$
\begin{aligned}
& \text { C1 }: \frac{p_{k, m}^{D} h_{k}^{D D}}{p_{m}^{C} h_{k, m}^{C D}+\sigma_{0}^{2}} \geq \frac{p_{k, m}^{D} h_{k, B}^{D B}+\sigma_{0}^{2}}{\sigma_{0}^{2}}, \forall k \in \mathcal{K}, m \in \mathcal{M}, \\
& \text { C2 }: \log _{2}\left(1+\frac{p_{m}^{C} h_{m, B}^{C B}}{\sum_{k=1}^{K} \pi_{k, m} p_{k, m}^{D} h_{k, B}^{D B}+\sigma_{0}^{2}}\right) \geq R_{m, \text { min }}^{C}, \forall m \in \mathcal{M}, \\
& \text { C3 : } \sum_{m=1}^{M} \pi_{k, m} p_{k, m}^{D} \leq p_{\mathrm{thr}}^{D}, \forall k \in \mathcal{K}, \\
& \text { C4 : } p_{k, m}^{D} \geq 0, \forall k \in \mathcal{K}, m \in \mathcal{M}, \\
& \text { C5 }: \sum_{k=1}^{K} \pi_{k, m} \leq 1, \forall m \in \mathcal{M}, \\
& \text { C6 }: \pi_{k, m} \in\{0,1\}, \forall k \in \mathcal{K}, m \in \mathcal{M} .
\end{aligned}
$$

where $R_{m, \min }^{C}$ is the required rate of CUs. C1 and C2 guarantee the positive system gain and the rate requirements of the CUs, respectively. $\mathrm{C} 2$ can be transformed as follows:

$$
\sum_{k=1}^{K} \pi_{k, m} p_{k, m}^{D} h_{k, B}^{D B} \leq \frac{p_{m}^{C} h_{m, B}^{C B}}{2^{R_{m, \text { min }}^{C}}-1}-\sigma_{0}^{2}, \forall k \in \mathcal{K} .
$$

C3 and C4 are the transmit power constraint of D2D pairs. C5 and C6 indicate that each CU's subcarrier resource can be reused by at most one D2D pair. 


\section{The Proposed Resource Allocation Scheme for D2D Communication}

The optimization problem formulated in (12) is an MINLP problem. In general, MINLP problems are known to be non-deterministic polynomial-time hard (NP-hard) and no efficient polynomial-time solutions exist, as the complexity may increase exponentially with the problem size. Moreover, it is not difficult to see that the problem (12) is a non-convex function since both $\pi_{k, m}$ and $p_{k, m}^{\mathrm{D}}$ are not jointly concave in $\left(\pi_{k, m}, p_{k, m}^{D}\right)$.

Despite this difficulty, this section focuses on solving the resource sharing optimization problem. To proceed, we use $\mathcal{M}_{k}=\left\{m \in \mathcal{M} \mid \pi_{k, m}=1\right\}$ to denote the set of the subcarriers which are assigned to the $k$ th D2D pair. All $\mathcal{M}_{1}, \mathcal{M}_{2}, \ldots, \mathcal{M}_{k}$ are subsets of $\{1,2, \ldots, M\}$. Then, problem (12) can be described as

$$
\begin{array}{cl}
\max _{\left\{p_{k, m}^{D}, \pi_{k, m}\right\}} & \sum_{k=1}^{K} \sum_{m=1}^{M} \pi_{k, m}\left[R_{k, m}^{D}\left(p_{k, m}^{D}\right)+R_{k, m}^{C}\left(p_{k, m}^{D}\right)-R_{k, m}^{C}\right] \\
\text { s.t. } & \text { C1 } \sim \text { C6 in }(12), \\
& \text { C7 }: \sum_{m \in \mathcal{M}_{k}} \pi_{k, m}=\left|\mathcal{M}_{k}\right|, \forall k \in \mathcal{K} .
\end{array}
$$

Constraint $\mathrm{C} 7$ represents the number of subcarriers that the D2D pair $k$ prepares for reusing.

Problem (14) is also a MINLP problem, which still needs a large amount of calculation to find the optimal solution. We can solve it efficiently by using a two-stage approach: subcarrier allocation and power assignment, which is verified by many existing similar problems [21,22]. Specifically, the original optimization problem (12) is decomposed into two individual sub-problems. The first problem is a system positive gain guaranteed subcarriers assignment, where we determine the optimal reuse subcarrier set $\mathcal{M}_{k}$ for D2D pairs. The second problem is power allocation, where we allocate the transmitted powers for D2D pairs to maximize the sum spectrum efficiency of D2D pairs and CUs in the heterogeneous network.

\subsection{Subcarrier Assignment}

In this subsection, we first need to solve the optimization problem for the case that one D2D pair $k$ shares the same subcarrier with the $\mathrm{CU} m$. Then we find the optimal matching of subcarriers between the D2D pairs and CUs by exploiting the feasible local optimum power allocation. Finally, we propose a heuristic subcarrier assignment algorithm to decide the subcarrier set $\mathcal{M}_{k}$ for each D2D pair.

Consider that the D2D pair $k$ shares the $m$ th subcarrier with CU $m$. To maximize the sum spectrum efficiency of $k$ and $m$, the considered problem can be formulated as

$$
\begin{aligned}
& \max _{p_{k, m}^{D}, k, m} R_{k, m}^{C D}\left(p_{k, m}^{D}\right) \triangleq R_{k, m}^{D}\left(p_{k, m}^{D}\right)+R_{k, m}^{C}\left(p_{k, m}^{D}\right) \\
& \text { s.t. C1 }: \frac{p_{k, m}^{D} h_{k}^{D D}}{p_{m}^{C} h_{k, m}^{C D}+\sigma_{0}^{2}} \geq \frac{p_{k, m}^{D} h_{k, B}^{D B}+\sigma_{0}^{2}}{\sigma_{0}^{2}}, \\
& \qquad \mathrm{C} 2: p_{k, m}^{D} h_{k, B}^{D B} \leq \frac{p_{m}^{C} h_{m, B}^{C B}}{2^{R_{m, m i n}^{C}}-1}-\sigma_{0}^{2}, \\
& \quad \mathrm{C} 3: 0 \leq p_{k, m}^{D} \leq P_{\mathrm{thr}}^{D} .
\end{aligned}
$$

According the constraints $\mathrm{C} 1$ and $\mathrm{C} 2$ in problem (15), we can easily get

$$
\left\{\begin{array}{l}
p_{k, m}^{D} \geq \frac{\sigma_{0}^{2}\left(p_{m}^{C} h_{k, m}^{C D}+\sigma_{0}^{2}\right)}{\sigma_{0}^{2}\left(h_{k}^{D D}-h_{k, B}^{D B}\right)-p_{m}^{C} h_{k, m}^{C D} h_{k, B}^{D B}} \triangleq p_{k, m}^{D \min 1}, \\
p_{k, m}^{D} \leq \frac{p_{m}^{C} h_{m, B}^{C B}}{h_{k, B}^{D B}\left(2^{\left.R_{m, \min }^{C}-1\right)}-\frac{\sigma_{0}^{2}}{h_{k, B}^{D B}} \triangleq p_{k, m}^{D} \max 1\right.} .
\end{array}\right.
$$

and we define

$$
p_{k, m}^{D \min } \triangleq \max \left\{0, \frac{\sigma_{0}^{2}\left(p_{m}^{C} h_{k, m}^{C D}+\sigma_{0}^{2}\right)}{\sigma_{0}^{2}\left(h_{k}^{D D}-h_{k, B}^{D B}\right)-p_{m}^{C} h_{k, m}^{C D} h_{k, B}^{D B}}\right\}
$$




$$
p_{k, m}^{D \max } \triangleq \min \left\{P_{\mathrm{thr}}^{D}, \frac{p_{m}^{C} h_{m, B}^{C B}}{h_{k, B}^{D B}\left(2^{R_{m, \min }^{C}}-1\right)}-\frac{\sigma_{0}^{2}}{h_{k, B}^{D B}}\right\}
$$

as the minimum and maximum possible powers of the D2D-Tx $k$ on the $m$ th subcarrier, respectively. Generally, $p_{k, m}^{D \text { min }} \leq p_{k, m}^{D \text { max }}$ should be satisfied. If $p_{k, m}^{D \min }>p_{k, m}^{D \text { max }}$, it means that D2D pair $k$ cannot share resources with CU $m$. Then (15) can be simplified to the following problem:

$$
\begin{array}{rl}
\max _{p_{k, m}^{D}, k, m} & U\left(p_{k, m}^{D}\right) \triangleq \log _{2}\left(p_{k, m}^{D} A_{1}+B_{1}\right) \\
& -\log _{2}\left(p_{m}^{C} h_{k, m}^{C D}+\sigma_{0}^{2}\right)+\log _{2}\left(p_{k, m}^{D} A_{2}+B_{2}\right) \\
& -\log _{2}\left(p_{k, m}^{D} A_{3}+B_{3}\right) \\
\text { s.t. } & \left\{\begin{array}{ll}
p_{k, m}^{D} \min \leq p_{k, m}^{D} \leq p_{k, m}^{D} \max \\
p_{k, m}^{D}=0
\end{array} \quad \text { if } \quad \begin{array}{c}
p_{k, m}^{D \min } \leq p_{k, m}^{D \text { max }}, \\
\text { otherwise, }
\end{array}\right.
\end{array}
$$

where

$$
\begin{aligned}
& A_{1}=h_{k}^{D D}, B_{1}=p_{m}^{C} h_{k, m}^{C D}+\sigma_{0}^{2}, \\
& A_{2}=A_{3}=h_{k, B}^{D B}, \\
& B_{2}=p_{m}^{C} h_{m, B}^{C B}+\sigma_{0}^{2}, B_{3}=\sigma_{0}^{2} .
\end{aligned}
$$

By taking the first order derivative of the $U\left(p_{k, m}^{\mathrm{D}}\right)$ versus $p_{k, m}^{\mathrm{D}}$ and setting the derivative to 0 , we can have

$$
\begin{aligned}
\frac{\partial U}{\partial p_{k, m}^{D}} & =\frac{1}{\ln 2} \cdot\left(\frac{1}{p_{k, m}^{D}+B_{1} / A_{1}}+\frac{1}{p_{k, m}^{D}+B_{2} / A_{2}}-\frac{1}{p_{k, m}^{D}+B_{3} / A_{3}}\right) \\
& =0 .
\end{aligned}
$$

With converting of the formula, (21) can be rewritten as follows:

$$
\frac{p_{k, m}^{D}{ }^{2}+B p_{k, m}^{D}+C}{\left(p_{k, m}^{D}+B_{1} / A_{1}\right)\left(p_{k, m}^{D}+B_{2} / A_{2}\right)\left(p_{k, m}^{D}+B_{3} / A_{3}\right)}=0 .
$$

where

$$
\begin{aligned}
& B=2 \frac{B_{3}}{A_{3}}, \\
& C=\left(\frac{B_{1}}{A_{1}}+\frac{B_{2}}{A_{2}}\right) \frac{B_{3}}{A_{3}}-\frac{B_{1}}{A_{1}} \frac{B_{2}}{A_{2}},
\end{aligned}
$$

Let $p_{k, m}^{D^{*}}$ be the optimal solution to the problem (15). Since $p_{k, m}^{D}>0$, the denominator of (22) is always positive. If $B^{2}-4 C<0, U\left(p_{k, m}^{D}\right)$ is monotonically increasing in the interval $\left[p_{k, m}^{D} \min , p_{k, m}^{D \max }\right]$. We can directly obtain $p_{k, m}^{D^{*}}=p_{k, m}^{D \max }$. If $B^{2}-4 C \geq 0$, the optimal solution to (15) can be obtained as follows:

$$
p_{k, m}^{D^{*}}=\left\{\begin{array}{lr}
{\left[\frac{-B \pm \sqrt{B^{2}-4 C}}{2}\right]_{p_{k, m}^{D \text { min }}}^{p_{k \text { max }}^{D}},} & p_{k, m}^{D \min } \leq p_{k, m}^{D \max } \\
0 . & p_{k, m}^{D \min }>p_{k, m}^{D \max }
\end{array}\right.
$$

where $[x]_{p_{k, m}^{D \text { min }}}^{p_{k \text { max }}^{D \text { max }}}$ represents the projection onto the interval $\left[p_{k, m}^{D \min }, p_{k, m}^{D \max }\right]$.

Next, we propose a heuristic greedy algorithm to find the optimal subcarriers assignment in order to maximize the sum spectrum efficiency. The operations of the assignment algorithm is shown in Algorithm 1. At the beginning of the allocation, the transmitters need to send some packets containing detection signals. Then, the obtained CSI at each UE (D2D or cellular receiver) would be feedback to the eNB so that various parameters can be initialized. By the previous definition, the sum spectrum efficiency brought by any D2D pair $k$ reusing any subcarrier $m$ can be roughly measured by expression $R_{k, m}^{C D}\left(p_{k, m}^{D}\right)$ in (15). Taking the maximum sum spectrum efficiency as a target, the algorithm selects optimal D2D pairs that have the maximum $R_{k, m}^{C D}\left(p_{k, m}^{D}\right)$ to reuse the $m$ th subcarrier. This procedure 
repeats until all subcarriers of the CUs have been assigned or cannot be assigned due to the maximum transmit power threshold being reached or because the transmit power is zero. If there are some subcarriers remaining that are still unshared by any D2D pair, we will try to allocate the remaining cellular resources to the D2D pairs that can further improve the spectrum efficiency gain.

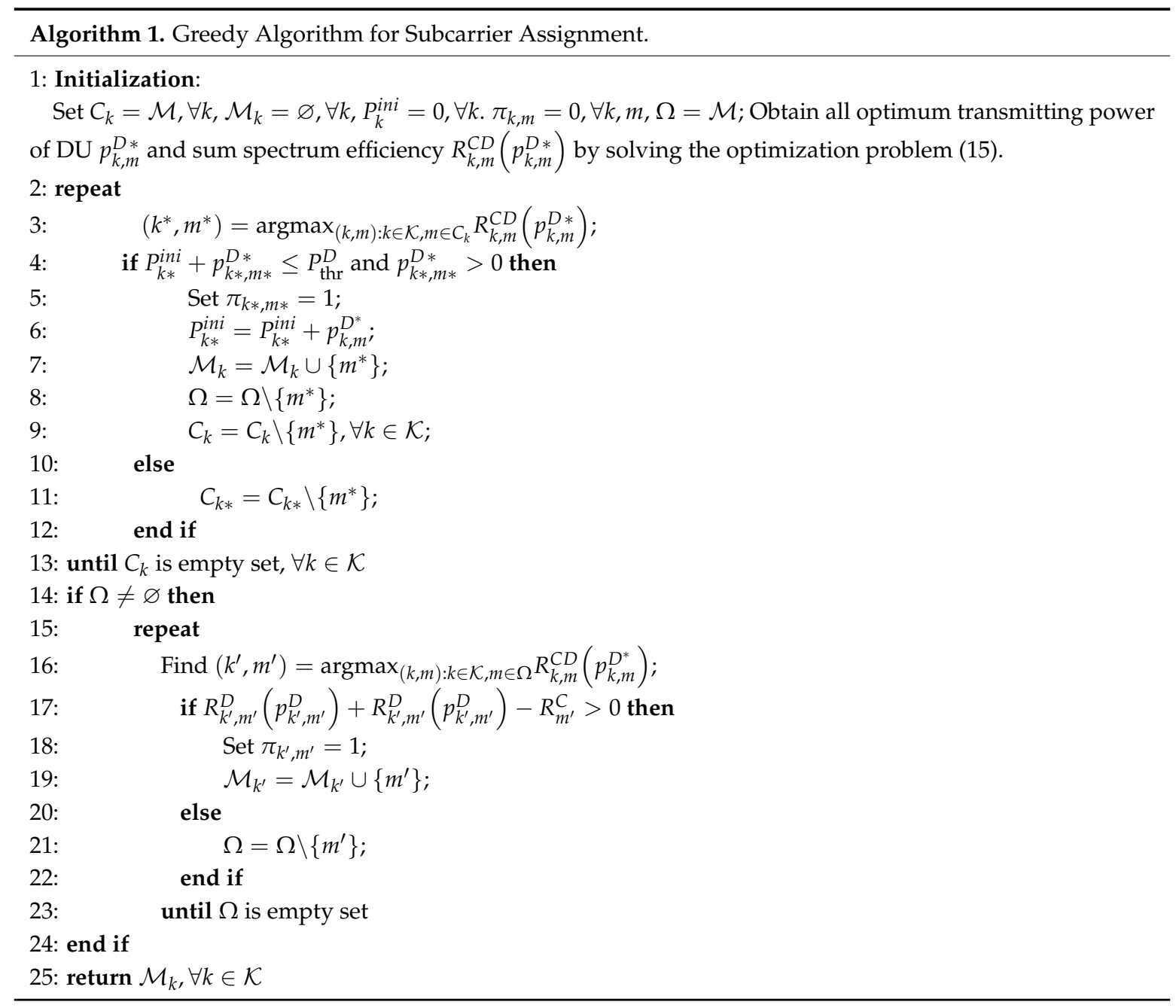

\subsection{Power Allocation with the Given D2D Pair}

Given the D2D pair $k$ and the subcarriers $m$ allocated to it, the binary variables $\pi_{k, m}$ in (12) are identified as 0 or 1 . In order to maximize the sum spectrum efficiency of overall heterogeneous network, the optimal transmission power allocated to each individual D2D pair $k$ should be found. Note that the set $\mathcal{M}_{k}$ represents subcarriers reused by the $k$ th D2D pair; the optimization problem of solving the optimal power allocation for the D2D pair $k$ can be expressed as:

$$
\begin{array}{lll}
\max _{p_{k, m}^{D}, k, m}^{\mathrm{Dax}} & \sum_{m \in \mathcal{M}_{k}} & {\left[R_{k, m}^{D}\left(p_{k, m}^{D}\right)+R_{k, m}^{C}\left(p_{k, m}^{D}\right)\right] \triangleq \sum_{m \in \mathcal{M}_{k}} Q\left(p_{k, m}^{D}\right)} \\
& \text { s.t. } & \mathrm{C} 1: p_{k, m}^{D} \geq p_{k, m}^{D} \min 1, \forall m \in \mathcal{M}_{k} \\
& \mathrm{C} 2: p_{k, m}^{D} \leq p_{k, m}^{D} \max 1, \forall m \in \mathcal{M}_{k}, \\
& \mathrm{C} 3: \sum_{m \in \mathcal{M}_{k}} p_{k, m}^{D} \leq P_{\mathrm{thr}}^{D} .
\end{array}
$$


According to $C 1$ in problem (12), we can easily get $B_{1} / A_{1} \leq B_{3} / A_{3}$. By taking the second order derivative of the $U\left(p_{k, m}^{D}\right)$ versus $p_{k, m}^{D}$, we have

$$
\begin{aligned}
\frac{\partial^{2} Q}{\partial p_{k, m}^{D}} & =\frac{1}{\ln 2} \cdot\left[-\frac{1}{\left(p_{k, m}^{D}+B_{1} / A_{1}\right)^{2}}-\frac{1}{\left(p_{k, m}^{D}+B_{2} / A_{2}\right)^{2}}\right. \\
& \left.+\frac{1}{\left(p_{k, m}^{D}+B_{3} / A_{3}\right)^{2}}\right] \leq 0 .
\end{aligned}
$$

indicating that $Q\left(p_{k, m}^{D}\right)$ is a concave function. Obviously, the optimization problem (25) is a standard convex optimization problem about variable $p_{k, m}^{D}$ and can be solved by Lagrangian Dual method [23]. The Lagrangian associated the problem (25) is

$$
\begin{aligned}
L\left(\left\{p_{k, m}^{D}\right\}, \lambda, \mu, v\right)=\sum_{m \in \mathcal{M}_{k}} & {\left[R_{k, m}^{D}\left(p_{k, m}^{D}\right)+R_{k, m}^{C}\left(p_{k, m}^{D}\right)\right] } \\
& +\sum_{m \in \mathcal{M}_{k}} \lambda_{m}\left(p_{k, m}^{D}-p_{k, m}^{D \min 1}\right)+\sum_{m \in \mathcal{M}_{k}} \mu_{m}\left(p_{k, m}^{D \max 1}-p_{k, m}^{D}\right) \\
& +v\left(P_{\mathrm{thr}}^{D}-\sum_{m \in \mathcal{M}_{k}} p_{k, m}^{D}\right)
\end{aligned}
$$

where $\lambda=\left\{\lambda_{m}\right\}, \mu=\left\{\mu_{m}\right\}, v$ are non-negative Lagrangian multipliers for the constraints $\mathrm{C} 1 \sim \mathrm{C} 3$ in (25). The dual problem of the optimization primal problem is defined as:

$$
\begin{array}{ll}
\min _{\{\lambda, \mu, v\}} & g(\lambda, \mu, v)=\max _{\left\{p_{k, m}^{D}\right\}} L\left(\left\{p_{k, m}^{D}\right\}, \lambda, \mu, v\right) \\
& \text { s.t. } \quad \lambda_{m} \geq 0, \mu_{m} \geq 0, v \geq 0
\end{array}
$$

For any $m \in \mathcal{M}_{k}, p_{k, m}^{D \text { opt }}$ is denoted as the optimal transmission power for D2D pair $k$. Then, the corresponding Karush-Kuhn-Tucker (KKT) condition is as follows:

$$
\begin{aligned}
& \frac{\partial g}{\partial p_{k, m}^{D}}= \frac{1}{\ln 2} \cdot\left(\frac{1}{p_{k, m}^{D}+B_{1} / A_{1}}+\frac{1}{p_{k, m}^{D}+B_{2} / A_{2}}\right. \\
&\left.-\frac{1}{p_{k, m}^{D}+B_{3} / A_{3}}\right)+\lambda_{m}^{*}-\mu_{m}^{*}-v^{*}=0, \\
& \lambda_{m}^{*}\left(p_{k, m}^{D o p t}-p_{k, m}^{D \min 1}\right)=0, \forall m \in \mathcal{M}_{k}, \\
& \mu_{m}^{*}\left(p_{k, m}^{D \max 1}-p_{k, m}^{D \text { opt }}\right)=0, \forall m \in \mathcal{M}_{k}, \\
& v^{*}\left(P_{\mathrm{thr}}^{D}-\sum_{m \in \mathcal{M}_{k}} p_{k, m}^{D o p t}\right)=0,
\end{aligned}
$$

where $\lambda_{m}^{*}, \mu_{m}^{*}$, and $v^{*}$ are the optimal dual points. If $p_{k, m}^{D \text { opt }}>p_{k, m}^{D \min 1}$ and $p_{k, m}^{D \text { opt }}<p_{k, m}^{D \max 1}$, we have $\lambda_{m}^{*}=0$ and $\mu_{m}^{*}=0$. Furthermore, due to the fact that $p_{k, m}^{D \text { opt }}$ are optimal, the tendency to pursue the power maximization requires that the equation $P_{\mathrm{thr}}^{D}-\sum_{m \in \mathcal{M}_{k}} p_{k, m}^{D \text { opt }}=0$ always follows. By substituting $\lambda_{m}^{*}=0, \mu_{m}^{*}=0$ into (29), the value of $v^{*}$ can be obtained as follows.

$$
\begin{aligned}
v^{*}= & \frac{1}{\ln 2}\left(\frac{1}{p_{k, m}^{D \text { opt }}+\frac{p_{m}^{C} h_{k, m}^{C D}+\sigma_{0}^{2}}{h_{k}^{D D}}}-\frac{1}{p_{k, m}^{D o p t}+\frac{\sigma_{0}^{2}}{h_{k, B}^{D B}}}\right. \\
& \left.+\frac{1}{p_{k, m}^{D \text { opt }}+\frac{p_{m}^{C} h_{m, B}^{C B}+\sigma_{0}^{2}}{h_{k, B}^{D B}}}\right) \triangleq f\left(p_{k, m}^{D o p t}\right)
\end{aligned}
$$

We develop a bisection algorithm to find the optimal value of $v$, i.e., $v^{*}$. The power allocation algorithm is shown in Algorithm 2, where $v^{u}$ and $v^{l}$ are the upper bound and lower bound of $v^{*}$, respectively. Starting from a feasible value of $v^{*}$, i.e., $v^{*}=\left(v^{u}+v^{l}\right) / 2$ in our algorithm, 
the corresponding $p_{k, m}^{D \text { opt }}$ can be calculated by solving the equivalent cubic Equation (33). Then $\sum_{m \in \mathcal{M}_{k}} p_{k, m}^{D \text { opt }}$ is worked out. Compare $\sum_{m \in \mathcal{M}_{k}} p_{k, m}^{D \text { opt }}$ with $P_{\mathrm{thr}}^{D}$, if $\sum_{m \in \mathcal{M}_{k}} p_{k, m}^{D \text { opt }}>P_{\mathrm{thr}}^{D}$, decrease $v^{*}$; if $\sum_{m \in \mathcal{M}_{k}} p_{k, m}^{D \text { opt }}<P_{\mathrm{thr}}^{D}$ increase $v^{*}$. The adjustment procedure runs until the condition $\sum_{m \in \mathcal{M}_{k}} p_{k, m}^{D \text { opt }}=P_{\mathrm{thr}}^{D}$ is satisfied or $v^{*}$ does not change.

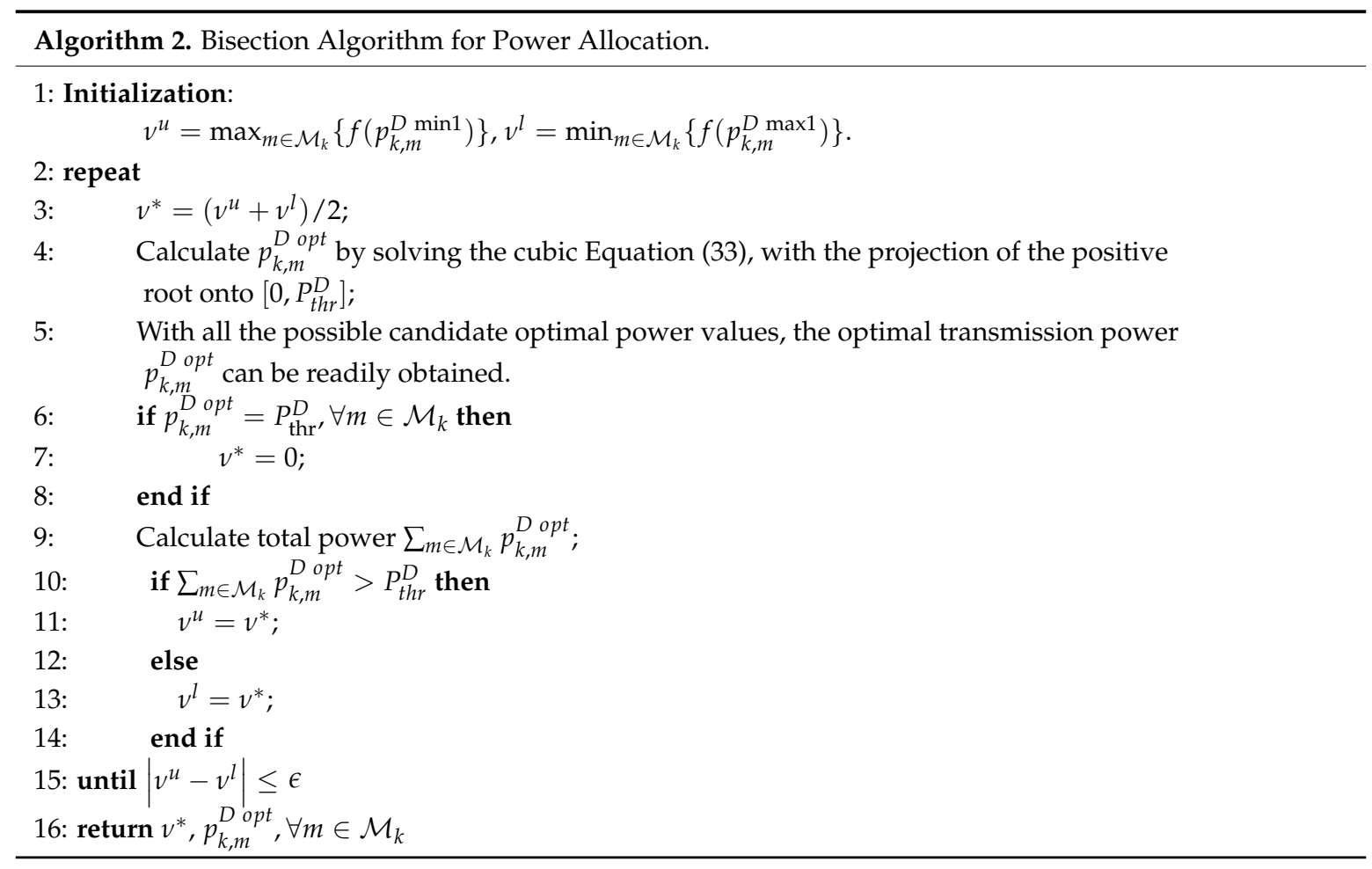

\subsection{Computational Complexity Analysis}

We analyze the complexity of our proposed algorithm in this subsection. In Algorithm 1, first we need to calculate all $p_{k, m}^{D^{*}}$ and $R_{k, m}^{C D}\left(p_{k, m}^{D^{*}}\right)$, then we search the optimal power allocation and decide the subcarrier set $\mathcal{M}_{k}$ for each D2D pair. The above two steps are irrelevant, and the complexity of both the two steps are $\mathcal{O}(K M)$. Finally, we try to allocate the remaining subcarriers to all the D2D pairs, where the complexity of the final step in Algorithm 1 is $\mathcal{O}(K \Omega),|\Omega| \leq|\mathcal{M}|$. Hence, the complexity of the subcarrier assignment algorithm is $\mathcal{O}(K M)$. In Algorithm 2, with ie as the accuracy requirement for the bisection algorithm, the complexity of the power allocation for the $k$ th D2D pair is $\mathcal{O}\left(\log _{2}(1 / \epsilon)\left|\mathcal{M}_{k}\right|\right)$. Therefore, the total complexity of our algorithm is $\mathcal{O}\left(\log _{2}(1 / \epsilon) K M\right)$. Obviously, our algorithm has polynomial complexity which has a linear relation with the scale of $K$ and $M$.

\section{Numerical Results}

In this section, we provide several simulation results to evaluate the performance of our proposed resource allocation scheme. Consider a single cell with a radius of $500 \mathrm{~m}$ where CUs and D2D pairs are uniformly and randomly distributed. Since the distance between D2D-Tx and D2D-Rx are relatively short, we assume all D2D pairs have the same transmitting distance. The main simulation parameters such as path loss models, shadowing standard deviation, subcarrier bandwidth, etc. are the same as these proposed in [24]. The related parameters used in the simulation experiments are summarized in Table 2. The tolerance $\epsilon$ is set to $10^{-5}$. 
Table 2. Main Simulation Parameters.

\begin{tabular}{cc}
\hline Parameter & Value \\
\hline Cellular layout & one isolated cellular cell \\
System area & $500 \mathrm{~m} \times 500 \mathrm{~m}$ \\
Maximum distance of D2D, $r$ & $20,30,40 \mathrm{~m}$ \\
Uplink subcarrier bandwidth & $180 \mathrm{kHz}$ \\
Noise spectral density & $-174 \mathrm{dBm} / \mathrm{Hz}$ \\
Path loss model for cellular links & $128.1+37.6 \log 10(\mathrm{~d}[\mathrm{~km}])$ \\
Path loss model for D2D links & $148+40 \log 10(\mathrm{~d}[\mathrm{~km}])$ \\
Shadowing standard deviation & $10 \mathrm{~dB}$ for cellular links \\
Maximum Tx power of CU & $12 \mathrm{~dB}$ for D2D links \\
Maximum Tx power of D2D & $20 \mathrm{dBm}$ \\
Minimum Rate of CU & $-10 \mathrm{dBm} \sim 20 \mathrm{dBm}$ \\
Number of CUs, $M$ & $0 \mathrm{bps} / \mathrm{Hz} \sim 6 \mathrm{bps} / \mathrm{Hz}$ \\
Number of D2D pairs, $K$ & $10 \sim 30$ \\
\hline
\end{tabular}

We compare the proposed scheme with three other schemes. Scheme 1 is proposed in [9], where throughput of multiple D2D pairs is maximized by using the Hungarian method. Scheme 2 randomly selects the subcarrier for D2D pairs to reuse and performs power allocation optimization to maximize the total achievable rate of the D2D pairs. Scheme 1 and Scheme 2 can only be applied to the case that each D2D pair could reuse only one single cellular resource. Scheme 3 is developed in [17], where only one D2D pair has the authority to use all subcarriers.

In Figure 2, we compare the spectrum efficiency of CUs and DUs versus the different transmit power budgets for D2D pairs, where $K=20, M=30, P_{\mathrm{thr}}^{D}=20 \mathrm{dBm}$, and $R_{m, \min }^{C}=6 \mathrm{bps} / \mathrm{Hz}$. From the figure, the spectrum efficiency of D2D pairs increases dramatically with the D2D transmit power budget; on the contrary, since greater power of DUs brings more serious interference to CUs, the spectrum efficiency of CUs decreases with the increasing of D2D transmit power budget. When the D2D transmit power is small, for example, $P_{\mathrm{thr}}^{D} \leq 10 \mathrm{dBm}$, the movement of D2D transmit power has great influence on the spectrum efficiencies of DUs and CUs, which have great improved and reduced tendency, respectively. When the D2D transmit power budget is increased to some degree, it would not improve the spectrum efficiency of DUs effectively, the growth in spectrum efficiency of DUs tends to saturation. Moreover, we can see that as the distance of D2D decreases, the spectrum efficiency of DUs increases significantly, while the spectrum efficiency of CUs reduces slightly. The reason is that the smaller the D2D distance, the better the D2D channel conditions and the higher rate in D2D communications. Meanwhile, with the decrease of D2D distance (from $40 \mathrm{~m}$ to $20 \mathrm{~m}$ ), the value range of D2D optimal transmit power is extended, which allows the D2D pairs to have more chance to reuse more subcarriers and causes more interference to CUs. While the D2D transmit power budget is large enough, the effect of interference caused by D2D transmit power on spectrum efficiency is more significant than that of D2D distance, and the curve becomes smooth. In addition, the increment of DUs in spectrum efficiency is greater than the decrement of CUs with the decrease of D2D distance, which implies that limiting the maximum communication distance of D2D can effectively improve the sum spectrum efficiency of the heterogeneous network. 


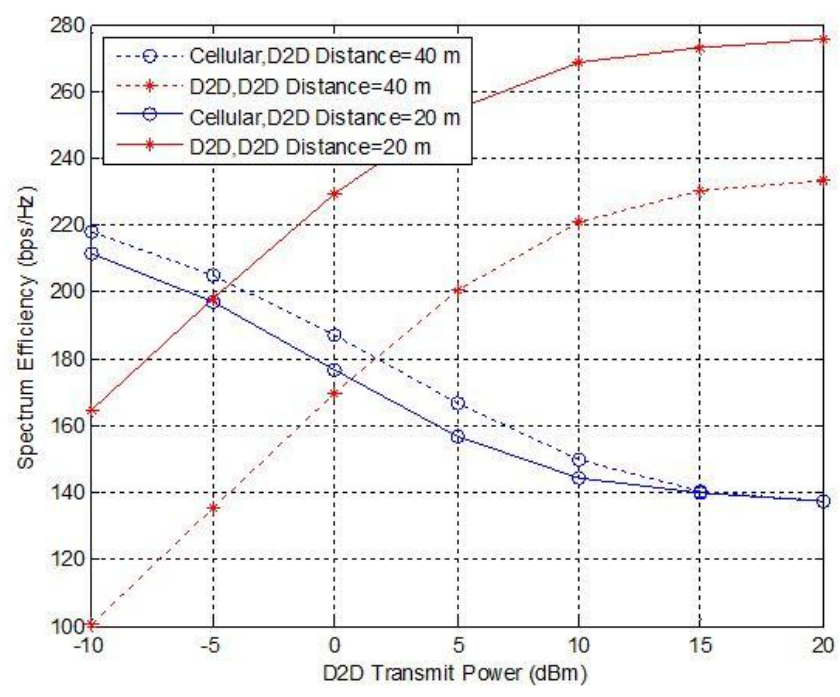

Figure 2. The spectrum efficiencies of the D2D pairs and CUs versus the transmit power budget.

Figure 3 shows the sum spectrum efficiency of the heterogeneous network with different number of CUs, where $K=8, r=30 \mathrm{~m}, P_{\mathrm{thr}}^{D}=20 \mathrm{dBm}$, and $R_{m \text {, min }}^{C}=6 \mathrm{bps} / \mathrm{Hz}$. Note that we assume the number of CUs equals the number of subcarriers, since each subcarrier is occupied by a CU. We observe that the sum spectrum efficiency goes up with the number of CUs increasing in all compared schemes, which indicates that more DUs contribute to a higher system sum spectrum efficiency. From the figure, our proposed scheme, i.e., the scheme of reusing multiple subcarriers, performs much better than the other three schemes. In particular, when the number of CUs is relatively small, for example, $M=10$, the performance of the proposed scheme is close to Scheme 1 . With the increasing number of CUs, the absolute performance gap expands. The relative performance gap when there are 30 CUs is about $19 \%$. This is due to the fact that each D2D pair can use more subcarriers as the number of CUs increases in the scenario of reusing multiple CUs' subcarriers. Obviously, Scheme 3 has the lowest sum spectrum efficiency. This is due to the fact that only one D2D pair gets involved in resource sharing; as a result, there may be many cellular subcarriers that may not be reused, leading to inefficiency in resource utilization.

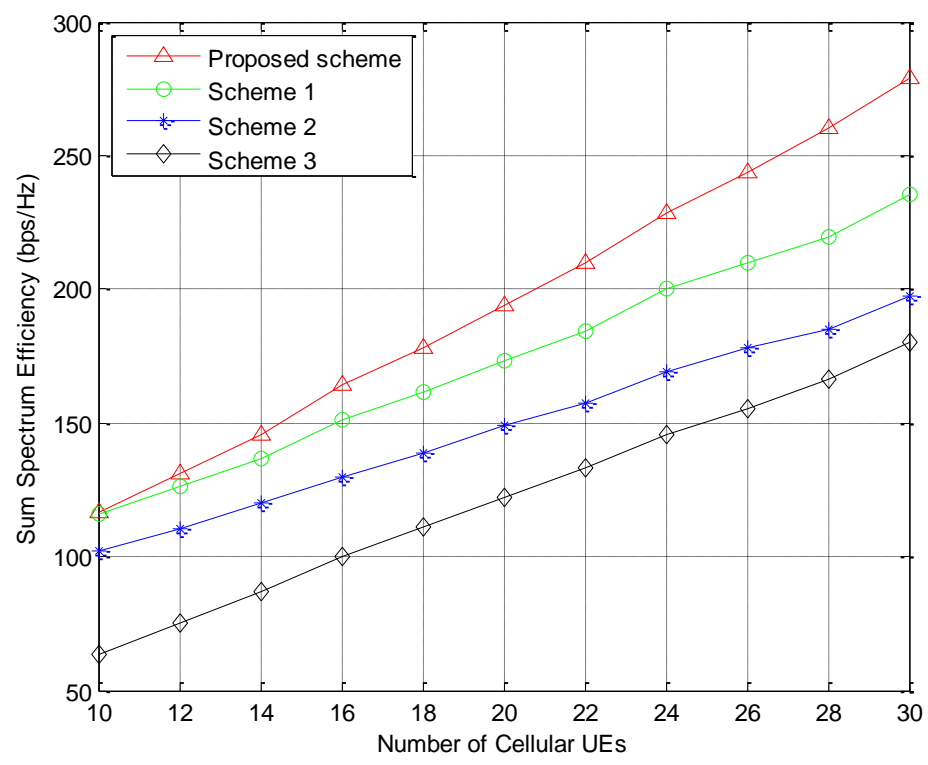

Figure 3. The sum spectrum efficiency versus the number of CUs $M$. 
The sum spectrum efficiency of the heterogeneous network with different D2D transmit power budget is illustrated in Figure 4, where $K=20, M=30, r=30 \mathrm{~m}$, and $R_{m, \min }^{C}=6 \mathrm{bps} / \mathrm{Hz}$. Intuitively, the sum spectrum efficiency rises with the increase of D2D transmit power budget. Since the total power can be allocated to multiple subcarriers of multiple D2D pairs, our proposed sharing scheme exhibits the best performance and the largest increases in sum spectrum efficiency. In addition, the sum spectrum efficiency increases rapidly in the lower D2D transmission power case. When $P_{\mathrm{thr}}^{D} \geq 10 \mathrm{dBm}$, the growth curve becomes smooth, which implies that the sum spectrum efficiency starts to saturate and the D2D transmit power does not have a major impact on the performance improvement. Figure 5 shows the sum spectrum efficiency with different rate requirement of the CUs, where $K=20, M=30$, $r=30 \mathrm{~m}$, and $P_{\mathrm{thr}}^{D}=20 \mathrm{dBm}$. Similarly, it is obvious that the proposed scheme performs much better than the other three. Moreover, with the increasing rate requirements of CUs, the sum spectrum efficiency of the heterogeneous network decreases slightly. With higher rate requirements, more transmission powers for CUs are needed to meet the required QoS, and thus more D2D pairs that share the same subcarriers with these CUs are restricted, resulting in that only the D2D pairs which generate low interference to the eNB can reuse the subcarriers.

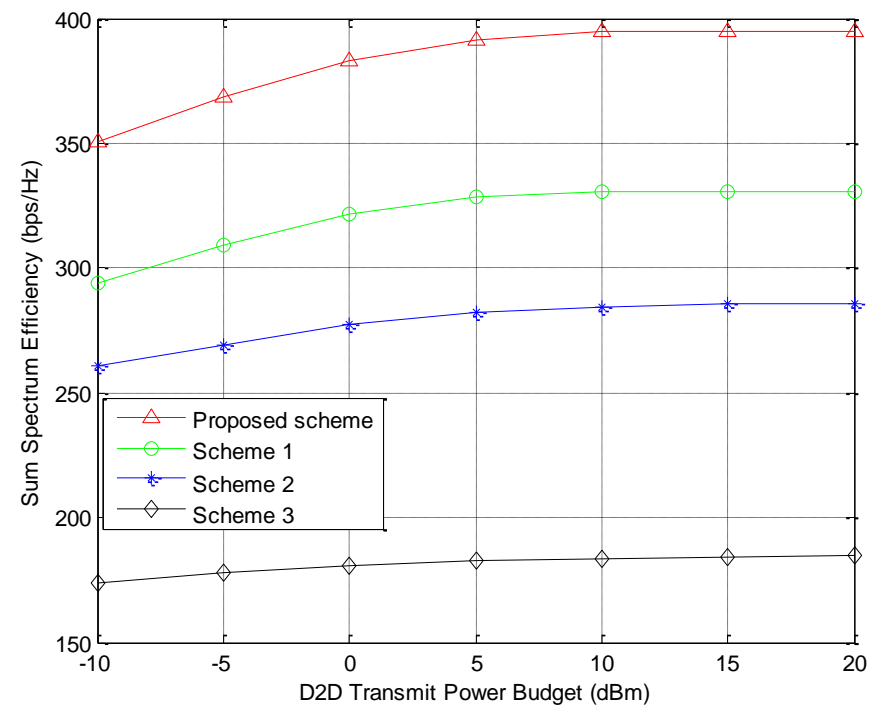

Figure 4. The sum spectrum efficiency versus the transmit power budget.

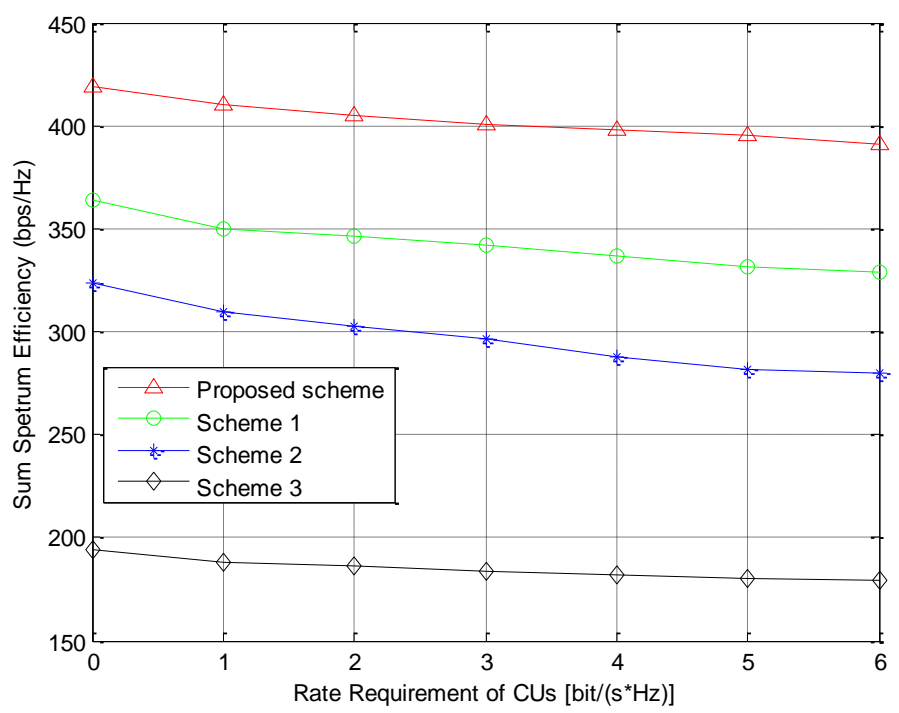

Figure 5. The sum spectrum efficiency versus the rate requirements of CUs. 
Figure 6 plots the sum spectrum efficiency of the heterogeneous network with different D2D pairs, where $M=30, r=30 \mathrm{~m}, P_{\mathrm{thr}}^{D}=20 \mathrm{dBm}$, and $R_{m, \text { min }}^{C}=6 \mathrm{bps} / \mathrm{Hz}$. We observe that with the increase of D2D pairs, the proposed scheme shows a trend of rapid growth in sum spectrum efficiency and outperforms other schemes, whereas the performance of Scheme 3 remains nearly unchanged. Since the performance of D2D communication on spectrum efficiency is far better than that of traditional cellular communication, multiple D2D pairs reusing multiple subcarriers can achieve a much higher throughput than reusing only single subcarrier (Scheme 1 and Scheme 2), or a single D2D pair reusing multiple subcarriers (Scheme 3). We also compare our proposed sharing scheme with Scheme 1 and Scheme 2 on the performance of average D2D spectrum efficiency. From Figure 7, It is obviously that the average D2D spectrum efficiency decreases with the increasing number of D2D pairs. The reason is that with more D2D pairs entering the network, the average available subcarrier per D2D pair reuses is fewer, resulting in a performance penalty. In addition, the performance degradation of the proposed scheme is more significant, which indicates that the performance of the proposed scheme is more sensitive to the number of available subcarriers.

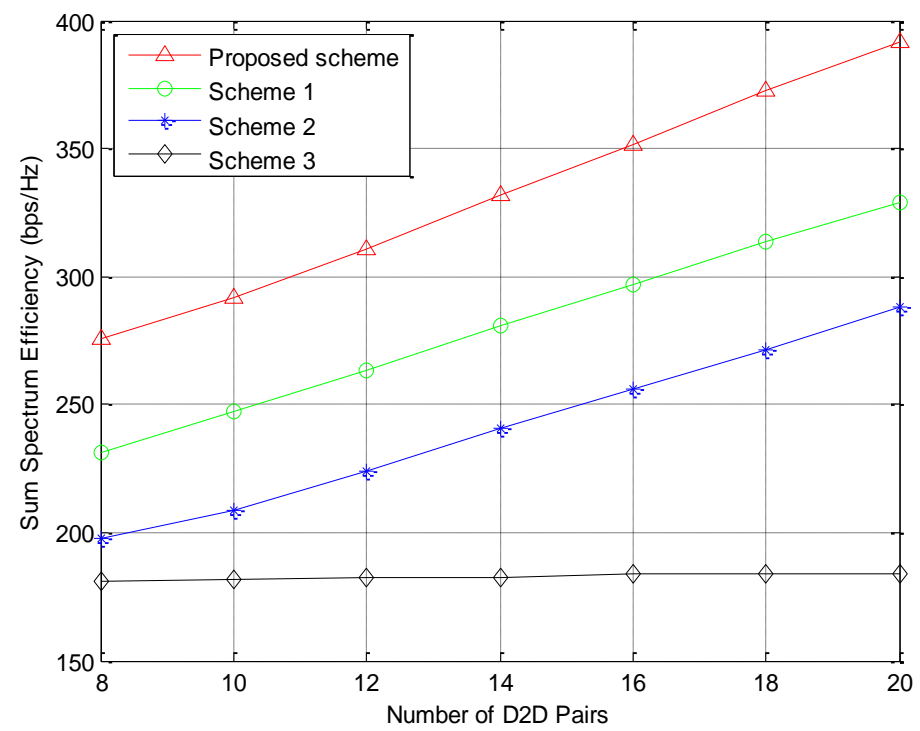

Figure 6. The sum spectrum efficiency versus the number of D2D pairs $K$.

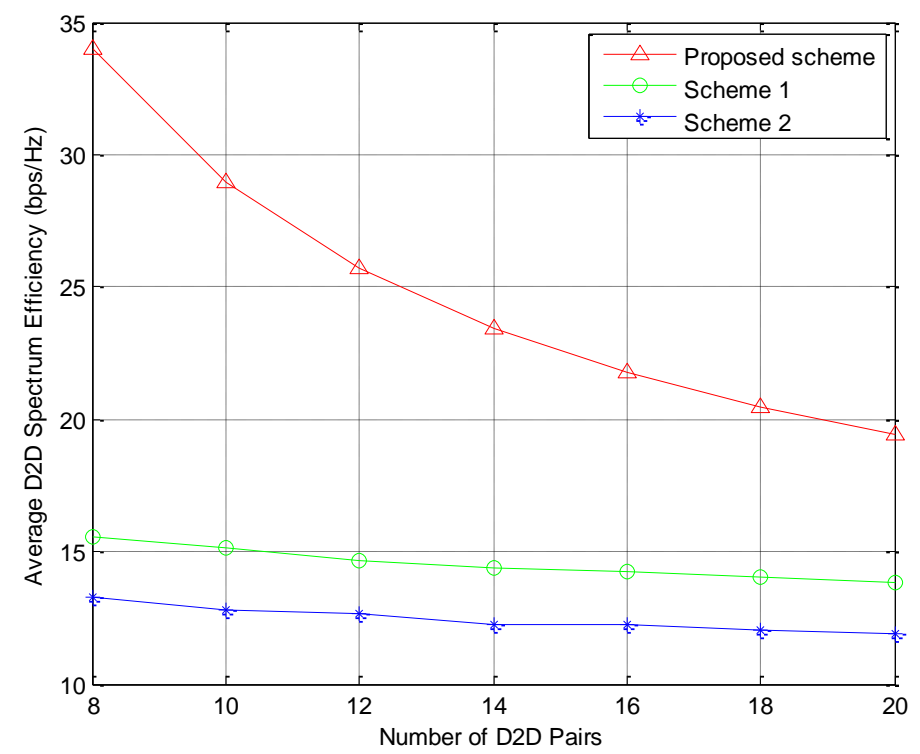

Figure 7. The average D2D spectrum efficiency versus the number of D2D pairs $K$. 


\section{Conclusions}

In this paper, a resource allocation scheme was studied for a multi-D2D communications underlaying cellular network, where each D2D pair can share subcarrier resources with multiple CUs. We formulated an optimization problem which maximized the sum spectrum efficiency of the heterogeneous network while guaranteeing a positive system gain and the rate requirement of CUs. A heuristic greedy algorithm was designed to make the subcarrier matching between CUs and D2D based on maximum sum spectrum efficiency, and then an efficient power allocation algorithm for each individual D2D pairs was developed based on the Lagrangian dual method. The simulation results showed that our proposed scheme can significantly improve the sum spectrum efficiency of the heterogeneous networks.

Acknowledgments: This work is sponsored by National Natural Science Foundation of China with Grant No. 61373042, and by the Natural Science Foundation of Hubei Province under Grant 2014CFB880.

Author Contributions: Yong Zhang and Fangmin Li initiated and discussed the research problem; Yong Zhang conceived and developed the methods; Yong Zhang and Mohammed Abdulaziz Aide Al-qaness performed the experiments and prepared the figures; Yong Zhang and Xidao Luan analyzed the data; Yong Zhang prepared and wrote the paper.

Conflicts of Interest: The authors declare no conflict of interest.

\section{References}

1. Doppler, K.; Rinne, M.; Wijting, C.; Ribeiro, C.; Hugl, K. Deviceto-device communication as an underlay to LTE-Advanced networks. IEEE Commun. Mag. 2009, 47, 42-49. [CrossRef]

2. Phunchongharn, P.; Hossain, E.; Kim, D.I. Resource allocation for device-to-device communications underlaying LTE-Advanced networks. IEEE Wirel. Commun. 2013, 20, 91-100. [CrossRef]

3. Fodor, G.; Dahlman, E.; Mildh, G.; Parkvall, S.; Reider, N.; Miklos, G.; Turanyi, Z. Design aspects of network assisted device-to-device communications. IEEE Commun. Mag. 2012, 50, 170-177. [CrossRef]

4. Lin, X.; Andrews, J.; Ghosh, A.; Ratasuk, R. An overview of 3GPP device-to-device proximity services. IEEE Wirel. Commun. 2014, 52, 40-48. [CrossRef]

5. Min, H.; Lee, J.; Park, S.; Hong, D. Capacity enhancement using an interference limited area for device-to-device uplink underlaying cellular networks. IEEE Trans. Wirel. Commun. 2011, 10, 3995-4000. [CrossRef]

6. Yu, C.H.; Doppler, K.; Ribeiro, C.; Tirkkonen, O. Resource sharing optimization for device-to-device communication underlaying cellular networks. IEEE Trans. Wirel. Commun. 2011, 10, 2752-2763.

7. Pei, Y.; Liang, Y.C. Resource allocation for device-to-device communications overlaying two-way cellular networks. IEEE Trans. Wirel. Commun. 2013, 12, 3611-3621. [CrossRef]

8. Han, T.; Yin, R.; Xu, Y.; Yu, G. Uplink channel reusing selection optimization for device-to-device communication underlaying cellular networks. In Proceedings of the 2012 IEEE 23rd International Symposium on Personal Indoor and Mobile Radio Communications (PIMRC), Sydney, Australia, 9-12 September 2012; pp. 559-564.

9. Feng, D.; Lu, L.; Yi, Y.W.; Li, G.; Feng, G.; Li, S. Device-to-device communications underlaying cellular networks. IEEE Trans. Wirel. Commun. 2013, 61, 3541-3551. [CrossRef]

10. Yu, G.; Xu, L.; Feng, D.; Yin, R.; Li, G.Y.; Jiang, Y. Joint mode selection and resource allocation for device-to-device communications. IEEE Trans. Wirel. Commun. 2014, 62, 3814-3824. [CrossRef]

11. Jung, M.; Hwang, K.; Choi, S. Joint mode selection and power allocation scheme for power-efficient device-to-device (D2D) communication. In Proceedings of the IEEE Vehicular Technology Conference (VTC Spring), Yokohama, Japan, 6-9 May 2012; pp. 1-5.

12. Yin, R.; Yu, G.; Zhang, H.; Zhang, Z.; Li, G.Y. Pricing-based interference coordination for D2D communications in cellular networks. IEEE Trans. Wirel. Commun. 2015, 14, 1519-1532. [CrossRef]

13. Wu, D.; Cai, Y.; Hu, R.; Qian, Y. Dynamic distributed resource sharing for mobile D2D communications. IEEE Trans. Wirel. Commun. 2015, 14, 5417-5429. [CrossRef]

14. Ng, D.W.K.; Lo, E.S.; Schober, R. Energy-Efficient Resource Allocation in Multi-Cell OFDMA Systems with Limited Backhaul Capacity. IEEE Trans. Wirel. Commun. 2012, 11, 3618-3631. [CrossRef] 
15. Ng, D.W.K.; Lo, E.S.; Schober, R. Wireless Information and Power Transfer: Energy Efficiency Optimization in OFDMA Systems. IEEE Trans. Wirel. Commun. 2013, 12, 6352-6370. [CrossRef]

16. Wang, B.; Chen, L.; Chen, X.; Zhang, X.; Yang, D. Resource allocation optimization for device-to-device communication underlaying cellular networks. In Proceedings of the IEEE Vehicular Technology Conference (VTC Spring), Budapest, Hungary, 15-18 May 2011; pp. 1-6.

17. Wang, J.; Zhu, D.; Zhao, C.; Li, J.; Lei, M. Resource sharing of underlaying device-to-device and uplink cellular communications. IEEE Commun. Lett. 2013, 17, 1148-1151. [CrossRef]

18. Zhu, H.; Wang, J. Chunk-based resource allocation in OFDMA systems-Part I: Chunk allocation. IEEE Trans. Commun. 2009, 57, 2734-2744.

19. Zhu, H.; Wang, J. Chunk-based resource allocation in OFDMA systems-Part II: Joint chunk, power and bit allocation. IEEE Trans. Commun. 2012, 60, 499-509. [CrossRef]

20. Hu, R.Q.; Qian, Y. An energy efficient and spectrum efficient wireless heterogeneous network framework for 5G systems. IEEE Commun. Mag. 2014, 52, 94-101. [CrossRef]

21. Wang, S.; Ge, M.; Wang, C. Efficient resource allocation for cognitive radio networks with cooperative relays. IEEE J. Sel. Areas Commun. 2013, 31, 2432-2441. [CrossRef]

22. Wang, S.; Ge, M.; Zhao, W. Energy-efficient resource allocation for OFDM-based cognitive radio networks. IEEE Trans. Wirel. Commun. 2013, 61, 3181-3191. [CrossRef]

23. Boyd, S.; Vandenberghe, L. Convex Optimization; Cambridge University Press: New York, NY, USA, 2004.

24. 3GPP. 3rd Generation Partnership Project; Technical Specification Group Radio Access Network; Further Advancements for E-UTRA Physical Layer Aspects (TR 36.814); Technical Report; 3GPP Organizational Partners' Publications Offices: Sophia Antipolis, France, 2010.

(C) 2017 by the authors; licensee MDPI, Basel, Switzerland. This article is an open access article distributed under the terms and conditions of the Creative Commons Attribution (CC BY) license (http://creativecommons.org/licenses/by/4.0/). 